\title{
Malignant Phyllodes Tumor in an Adolescent Female: A Rare Case Report and Review of the Literature
}

\author{
Gabriel S. Makar $\mathbb{D}^{1},{ }^{1}$ Michael Makar ${ }^{D},^{2}$ Joanna Ghobrial, ${ }^{3}$ Kathryn Bush, ${ }^{1}$ \\ Ryan Allen Gruner, ${ }^{4}$ and Thomas Holdbrook ${ }^{5}{ }^{5}$ \\ ${ }^{1}$ Cooper Medical School of Rowan University, 401 Broadway Ave, Camden, NJ 08103, USA \\ ${ }^{2}$ Robert Wood Johnson University Hospital, 1 Robert Wood Johnson Pl, New Brunswick, NJ 08901, USA \\ ${ }^{3}$ AT Still University School of Osteopathic Medicine, 5850 E Still Cir, Mesa, AZ 85206, USA \\ ${ }^{4}$ Cooper University Hospital, Department of Surgery, 1 Cooper Plaza, Camden, NJ 08103, USA \\ ${ }^{5}$ Cooper University Hospital, Department of Pathology, 1 Cooper Plaza, Camden, NJ 08103, USA
}

Correspondence should be addressed to Gabriel S. Makar; makarg3@rowan.edu

Received 15 January 2020; Accepted 13 February 2020; Published 28 February 2020

Academic Editor: Jose I. Mayordomo

Copyright (c) 2020 Gabriel S. Makar et al. This is an open access article distributed under the Creative Commons Attribution License, which permits unrestricted use, distribution, and reproduction in any medium, provided the original work is properly cited.

\begin{abstract}
Primary breast neoplasms are rare in adolescent females, most of which are benign. Phyllodes tumors constitute a remarkably small subset of breast neoplasms (0.3-0.9\%) with malignant phyllodes tumors being even more uncommon. Malignant phyllodes tumors tend to progress rapidly though only $1.5 \%$ metastasize. They are also associated with a higher rate of recurrence than their benign counterparts, underlying the importance of adequate surgical margins. It is therefore imperative to be able to identify these tumors early allowing for prompt resection and close follow-up. Here, we present the rare case of a 17-year-old female presenting with a rapidly enlarging breast mass, which was ultimately found to be a malignant phyllodes tumor. We further performed a review of the literature to highlight only 22 other cases reported in adolescent females.
\end{abstract}

\section{Introduction}

Primary breast neoplasms are extremely rare in adolescent females and most are benign in nature. The incidence of primary breast tumors in females under the age of 20 is approximately 1 in one million [1]. Phyllodes tumors of the breast, previously known as cystosarcoma phyllodes, are rare fibroepithelial tumors that constitute approximately $0.3-0.5 \%$ of all breast neoplasms $[2,3]$. The incidence for these tumors is remarkably low with an estimated 2.1 per one million women with a higher prevalence among Latina whites [4]. They occur most commonly during the late fifth decade of life in females and are even more rarely do they occur in men $[5,6]$. Clinically, these tumors present as palpable, sometimes rapidly enlarging masses of the breast with a median size of approximately $4 \mathrm{~cm}$ [7]. Additionally, malignant phyllodes tumors are, on average, larger than their borderline or benign counterparts [6].
Malignant phyllodes tumors account for approximately $6.5-27 \%$ of all phyllodes tumors [5, 8-11]. Compared to benign and borderline phyllodes tumors, malignant tumors contain a higher rate of disease recurrence, decreased overall survival, and distant metastasis $[5,10]$. Although they are more aggressive, cause-specific survival for these patients was $91 \%, 89 \%$, and $89 \%$, at 5,10 , and 15 years, respectively [12]. The median age of diagnosis remains similar to benign phyllodes at around 50 years old [12].

Herein, we perform a review of the literature and present the rare case of an adolescent female with a malignant phyllodes tumor.

\section{Case Description}

A 17-year-old female presented to the emergency department (ED) regarding an enlarging right breast mass. She noticed the mass 1 week prior to presentation. It was associated with mild 


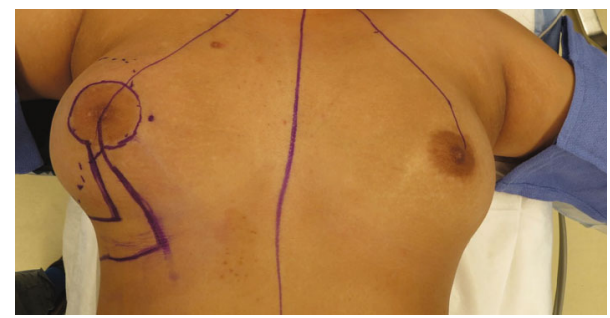

Figure 1: Preoperative image capturing prominence of tumor size and breast distortion relative to contralateral breast.

intermittent breast pain and severe periodic episodes of pain rated 10/10. No relieving or exacerbating factors were noted. The patient denied any redness or thickening, nipple discharge, nipple inversion, and any noticeable changes on the contralateral breast. Her breast sizes had not changed relative to one another. She had no previous history of breast biopsies or abnormal breast imaging. She denied additional symptoms including bone pain, rib pain, headache, and shortness of breath and was otherwise in her usual state of health. Her family history was significant only for a maternal aunt with ovarian cancer. The patient underwent menarche at the age of 12 . There was no history of use of exogenous hormones to date. Her last menstrual period was approximately 25 days prior to presentation to the ED. Physical exam revealed a large, nontender approximately $15 \times 11 \mathrm{~cm}$ mass occupying the majority of her right lateral breast (Figure 1). The left breast did not appear to have any masses, inflammatory skin changes, or nipple-areolar complex abnormalities. There was no axillary, infraclavicular, or supraclavicular lymphadenopathy appreciated on exam.

Breast ultrasound at the time of presentation revealed a $11.0 \times 7.2 \times 5.1 \mathrm{~cm}$ complex heterogeneous hypoechoic solid mass, which demonstrated mild internal vascularity on color Doppler flow. The mass was noncompressible with surrounding peripheral fluid. The patient underwent an ultrasound-guided core needle biopsy of the mass, which revealed a fibroepithelial tumor. Final pathology favored phyllodes tumor. The patient was also seen by plastic surgery regarding the anticipated large defect post resection of the tumor. Given the pathological findings, the patient underwent a wide right breast mass excision with a complex closure of the $10 \mathrm{~cm}$ wound. Pathologic examination revealed a $12.5 \mathrm{~cm}$ malignant phyllodes tumor (Figure 2). Histologic sections of the tumor showed the characteristic leaf-like architecture of phyllodes tumor with areas of prominent hypercellular stroma. The hypercellular stroma displayed marked nuclear pleomorphism and mitotic activity as well as areas of necrosis (Figure 3-5). The tumor was within $0.5 \mathrm{~mm}$ of the closest resection margin. Therefore, per the 2019 National Comprehensive Cancer Network guidelines, the patient was sent back to the operating room for reexcision lumpectomy to ensure full $1 \mathrm{~cm}$ margins around the malignant tumor. A lateral intercostal artery perforator flap was performed for appropriate wound coverage.

The patient has been doing well since the time of surgery with no current signs of metastasis or local recurrence. Chemotherapy was not currently indicated, and although

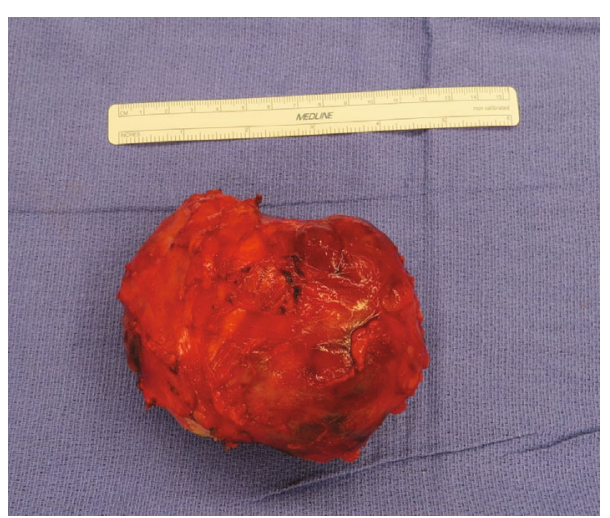

Figure 2: Intraoperative image of excised tumor measuring $12.5 \mathrm{~cm}$.

radiation therapy was recommended, the patient refused radiation and opted for close observation [13]. Workup for metastatic and recurrent disease has been negative to date.

We additionally performed a literature review of adolescent females, defined as those 18 years of age or younger, with a confirmed diagnosis of a malignant phyllodes tumor (Table 1). Our report relies predominantly on the review attained from Levêque et al. who reviewed 18 adolescent females with malignant phyllodes tumors and presented a patient of their own (total 19 patients) [14-29]. Our review includes three additional patients for a total of 22 affected adolescent females [30, 31].

\section{Discussion}

Primary breast neoplasms are uncommon among adolescent females and may be misdiagnosed as a physiologic breast mass, as these are more common in this demographic. Phyllodes tumors constitute a rare subset of breast neoplasms $(<1 \%)$, with a predilection for women in their late fifth to sixth decade of life and malignant phyllodes tumors account for an even smaller cohort (6.5-27\% of all phyllodes tumors). Moreover, malignant phyllodes tumors occur in a population comparable to benign tumors, with rare occurrences in the adolescent population. Our report adds to the scarce literature of adolescent females with malignant phyllodes tumors and provides an overview of these tumors in the adolescent female population.

Phyllodes tumors are fibroepithelial neoplasms of the breast, which can histologically resemble fibroadenomas but typically display distinct stromal hypercellularity, prominent intracanalicular growth pattern, and characteristic leaf-like architecture. The World Health Organization (WHO) classifies phyllodes tumors into benign, borderline, and malignant categories based on the degree of stromal hypercellularity, cytological atypia, mitotic activity appearance of tumor border, and stromal overgrowth [32, 33]. Table 2 contains the current WHO criteria for diagnosis of phyllodes tumors [34]. The intracanalicular growth pattern along with hypercellularity is essential in differentiating fibroadenomas from benign phyllodes tumors, which can at times be difficult. Additionally, progression of fibroadenoma to malignant phyllodes tumors is not unknown as seen in case number 20 (Table 1). In a review of 36 malignant 


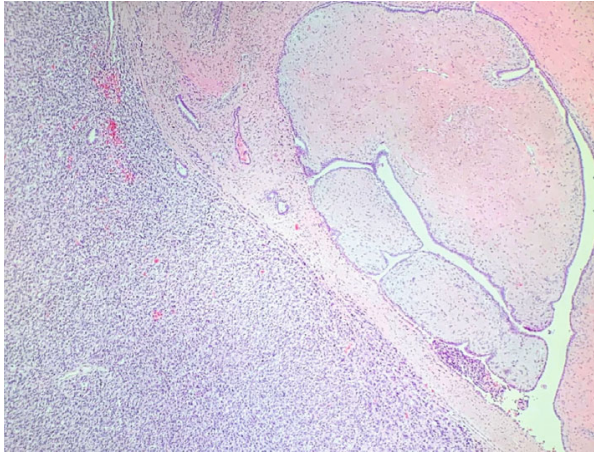

(a)

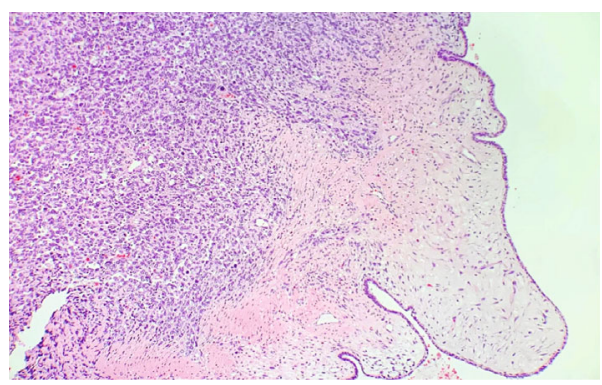

(b)

FIgURE 3: Malignant phyllodes tumor. ( $\mathrm{a}, \mathrm{b})$ The characteristic leaf-like architecture with epithelium lined clefts is present (right) next to prominent hypercellular stroma (left) (H\&E, $\times 40$ (a), $\times 100$ (b)).

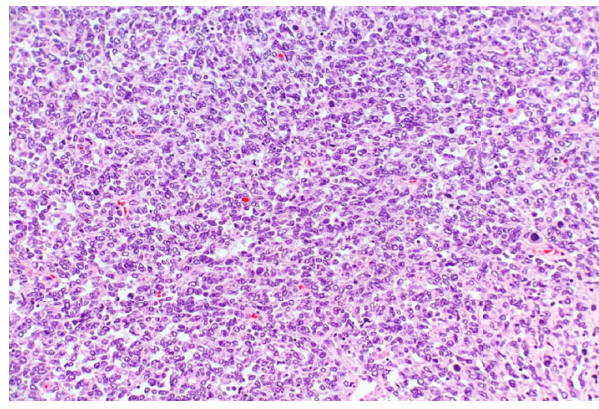

(a)

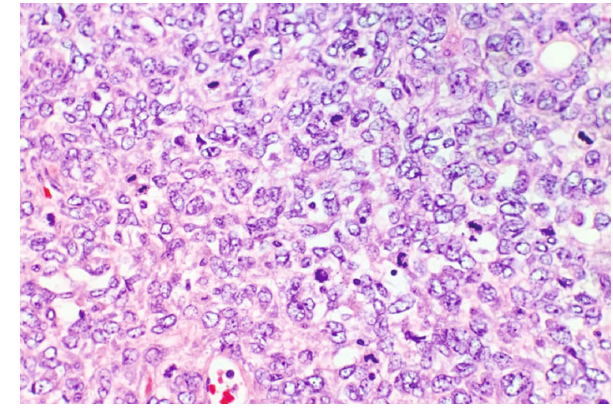

(b)

Figure 4: Malignant phyllodes tumor. (a, b) There is marked nuclear pleomorphism and mitotic activity (H\&E, $\times 200$ (a), $\times 400$ (b)).

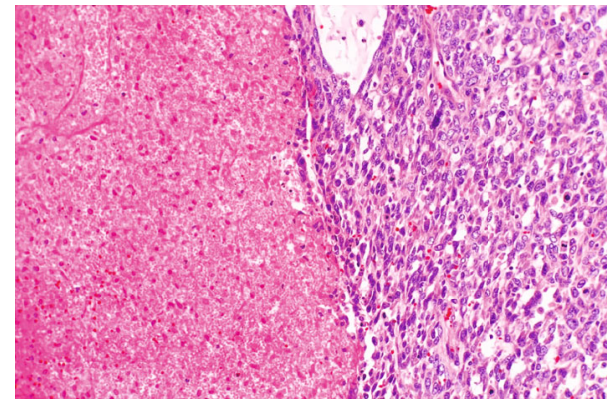

FIGURE 5: Malignant phyllodes tumor. An area of tumor cell necrosis (left) $(\mathrm{H} \& \mathrm{E}, \times 200)$.

phyllodes tumors, $11(30.6 \%)$ were given a diagnosis of primary fibroadenomas and experienced recurrence as malignant phyllodes tumors [35]. To properly capture these tumors, the growth rate must be observed closely. Studies suggest growth more than $20 \%$ in 6 months may be concerning for phyllodes tumor over fibroadenoma and constitutes surgical excision or biopsy [36, 37].

Formulating a proper diagnosis for patients presenting with breast masses can be stratified based on the patient's age. Less than $10 \%$ of phyllodes tumors occur in females younger than 20 years of age [15]. With malignant tumors also being tremendously rare in this population, there is a high likelihood of missing such tumors. These tumors can also be aggressive and rapidly progressive as seen in our case and previous reports [11, 15]. Ample detection of these tumors requires prompt biopsy, as was done in our case, to rule out such aggressive malignancies. After the diagnosis is made, appropriate negative margins must be established to prevent tumor recurrence. For patients with malignant phyllodes tumor, consensus remains that negative margins of 1 to $2 \mathrm{~cm}$ be obtained for optimal excision or simple mastectomy if unable to reach such margins [38].

Recurrence rates for benign, borderline, and malignant tumors vary vastly among studies. The literature, in concordance with the work done by the WHO, suggests that recurrence among benign, borderline, and malignant phyllodes tumors is $10-17 \%, 14-25 \%$, and $23-30 \%$, respectively [32]. Benign phyllodes tumors may be followed closely even with positive margins; however, malignant tumors must have confirmed negative margins given the high rate of recurrence and even death $(22 \%)$ [32, 39]. Adolescents are said to have a higher rate of recurrence compared to their adult counterparts and therefore should be observed closely with routine follow-up even in light of negative margins [21]. Studies suggest Asian women appear to be at a higher risk of recurrence than non-Asian's [40]. However, a study utilizing the Surveillance, Epidemiology, and End Results Program (SEER) database found that there was no difference in survival among 


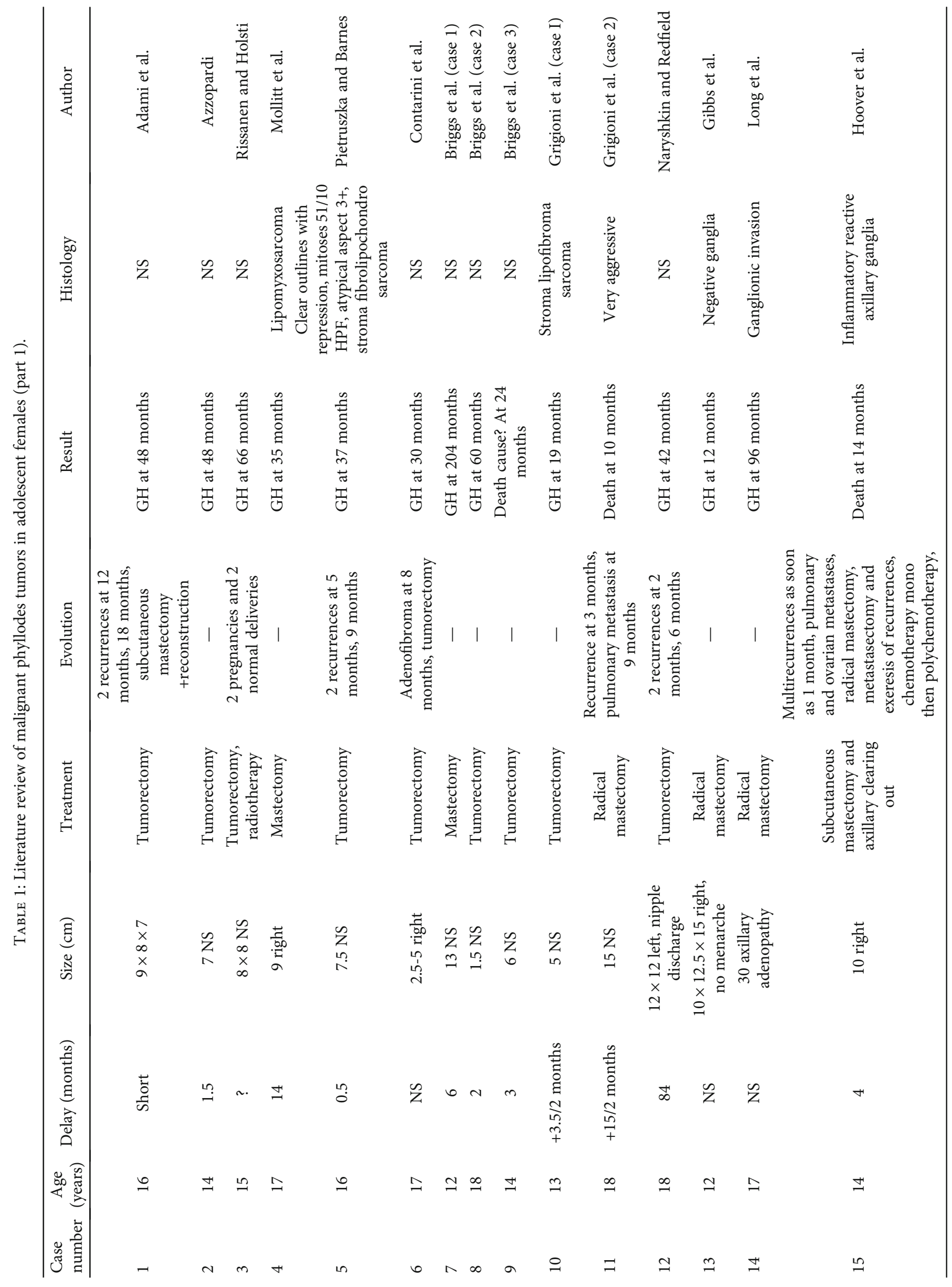




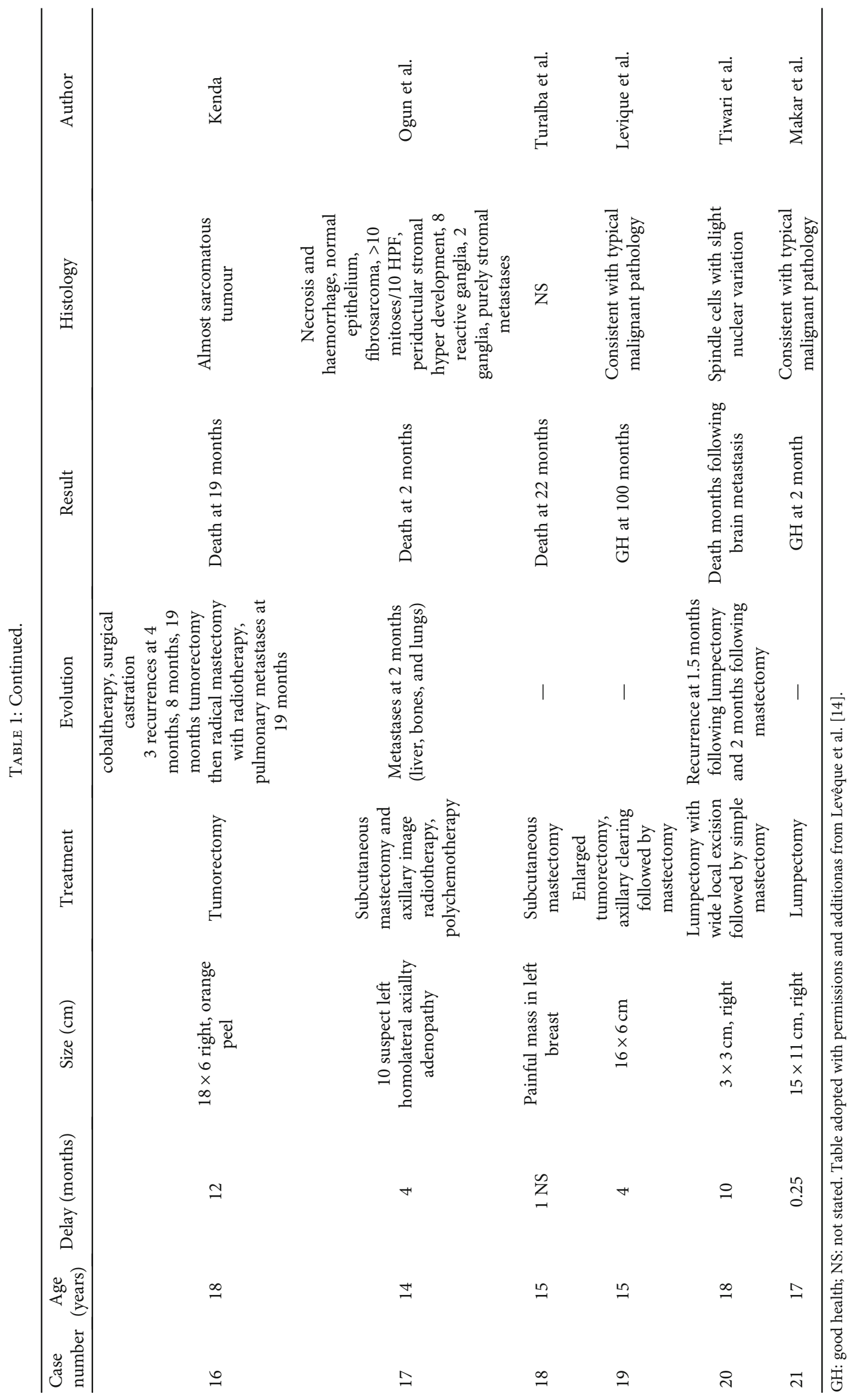


TABLE 2: Grading system for phyllodes tumors based on 2012 World Health Organization classification.

\begin{tabular}{lccc}
\hline Histologic features & Benign & Histological type & Borderline \\
\hline Stromal cellularity & Mild & Moderate & Malignant \\
Stromal atypia & Mild & Moderate & Marked \\
Mitosis (per 10 HPF) & $<5$ & 9 -may & Marked \\
Stromal overgrowth & Absent & Absent or focal & Present \\
Tumor margin & Clear & Clear or infiltration & Infiltration \\
\hline
\end{tabular}

HPF: high-power field. Table adopted with permissions from Zhang and Kleer [34].

white, black, and "other" ethnicities [41]. Further research is necessary to identify the true prevalence and incidence of disease among other ethnicities and in younger populations.

\section{Conclusion}

Given the unique and uncommon nature of primary breast tumors in adolescent females, appropriate assessment and management must be achieved to prevent misdiagnosing rare, aggressive tumors. It is necessary to be vigilant in the diagnosis and prompt management of rare tumors such as phyllodes tumors, due to the possibility of malignancy and rapid growth. Key features, such as large and rapidly growing mass, can aid in this diagnosis, but biopsy is necessary to characterize the suspect mass. We report the rare case of a rapidly growing malignant phyllodes tumor in an adolescent female.

\section{Disclosure}

This study was conducted at Cooper University Hospital, Department of Pathology 1 Cooper Plaza, Camden, NJ, 08103.

\section{Conflicts of Interest}

The authors declare that there is no conflict of interest regarding the publication of this paper.

\section{References}

[1] E. J. Lee, Y.-W. Chang, J. H. Oh, J. Hwang, S. S. Hong, and H.-J. Kim, "Breast lesions in children and adolescents: diagnosis and management," Korean Journal of Radiology, vol. 19, no. 5, pp. 978-991, 2018.

[2] M. D. Rowell, R. R. Perry, J. G. Hsiu, and S. C. Barranco, "Phyllodes tumors," American Journal of Surgery, vol. 165, no. 3, pp. 376-379, 1993.

[3] M. Reinfuss, J. Mituś, K. Duda, A. Stelmach, J. Ryś, and K. Smolak, "The treatment and prognosis of patients with phyllodes tumor of the breast: an analysis of 170 cases," Cancer, vol. 77, no. 5, pp. 910-916, 1996.

[4] L. Bernstein, D. Deapen, and R. K. Ross, "The descriptive epidemiology of malignant cystosarcoma phyllodes tumors of the breast," Cancer, vol. 71, no. 10, pp. 3020-3024, 1993.

[5] M. F. Rodrigues, P. T. Truong, E. C. McKevitt, L. M. Weir, M. A. Knowling, and E. S. Wai, "Phyllodes tumors of the breast: the British Columbia Cancer Agency experience," Cancer Radiothérapie, vol. 22, no. 2, pp. 112-119, 2018.

[6] O. Hamdy, G. A. Saleh, S. Raafat, A. M. Shebl, and A. Denewer, "Male breast huge malignant phyllodes," Chirurgia, vol. 114, no. 4, pp. 512-517, 2019.

[7] S. P. Mishra, S. K. Tiwary, M. Mishra, and A. K. Khanna, "Phyllodes tumor of breast: a review article," ISRN Surgery, vol. 2013, Article ID 361469, 10 pages, 2013.

[8] J. P. Bellocq, Ed.G. Magro, "Fibroepithelial tumours," in World Health Organization Classification of Tumours: Pathology and Genetics of Tumours of the Breast and Female Genital Organs, J. P. Bellocq, P. Devilee, and , Eds., pp. 99-103, IARC Press, Lyon, 2003.

[9] J. Chang, L. Denham, E. K. Dong, K. Malek, and S. S. Lum, "Trends in the diagnosis of phyllodes tumors and fibroadenomas before and after release of WHO classification standards," Annals of Surgical Oncology, vol. 25, no. 10, pp. 3088-3095, 2018.

[10] P. H. Tan, A. A. Thike, W. J. Tan et al., "Predicting clinical behaviour of breast phyllodes tumours: a nomogram based on histological criteria and surgical margins," Journal of Clinical Pathology, vol. 65, no. 1, pp. 69-76, 2012.

[11] A. Wada, N. Hayashi, F. Endo et al., "Repeat recurrence and malignant transition of phyllodes tumors of the breast," Breast Cancer, vol. 25, no. 6, pp. 736-741, 2018.

[12] O. K. Macdonald, C. M. Lee, J. D. Tward, C. D. Chappel, and D. K. Gaffney, "Malignant phyllodes tumor of the female breast: association of primary therapy with cause-specific survival from the surveillance, epidemiology, and end results (SEER) program," Cancer, vol. 107, no. 9, pp. 2127-2133, 2006.

[13] X. Chao, K. Chen, J. Zeng et al., "Adjuvant radiotherapy and chemotherapy for patients with breast phyllodes tumors: a systematic review and meta-analysis," BMC Cancer, vol. 19, no. 1, p. 372, 2019.

[14] J. Levêque, B. Meunier, E. Wattier, F. Burtin, J. Y. Grall, and J. Kerisit, "Malignant cystosarcomas phyllodes of the breast in adolescent females," European Journal of Obstetrics, Gynecology, and Reproductive Biology, vol. 54, no. 3, pp. 197-203, 1994.

[15] H. O. Adami, L. Hakelius, A. Rismten, and R. Willén, "Malignant, locally recurring cystosarcoma phyllodes in an adolescent female. A case report," Acta Chirurgica Scandinavica, vol. 150, no. 1, pp. 93-100, 1984.

[16] J. G. Azzopardi, Problems in breast pathology, WB Saunders, London, 1979.

[17] P. M. Rissanen and P. Holsti, "A retrospective study of sarcoma of the breast and the results of treatment," Oncology, vol. 22 , no. 4 , pp. $258-268,1968$. 
[18] D. L. Mollitt, E. S. Golladay, E. S. Gloster, and J. F. Jimenez, "Cystosarcoma phylloides in the adolescent female," Journal of Pediatric Surgery, vol. 22, no. 10, pp. 907-910, 1987.

[19] M. Pietruszka and L. Barnes, "Cystosarcoma phyllodes: a clinicopathologic analysis of 42 cases," Cancer, vol. 41, no. 5, pp. 1974-1983, 1978.

[20] O. Contarini, L. F. Urdaneta, W. Hagan, and S. E. Stephenson Jr., "Cystosarcoma phylloides of the breast: a new therapeutic proposal," The American Surgeon, vol. 48, no. 4, pp. 157$166,1982$.

[21] R. M. Briggs, M. Walters, and D. Rosenthal, "Cystosarcoma phylloides in adolescent female patients," The American Journal of Surgery, vol. 146, no. 6, pp. 712-714, 1983.

[22] W. F. Grigioni, D. Santini, A. Grassigli et al., "A clinicopathologic study of cystosarcoma phyllodes: 22 cases report," Archives D'Anatomie et de Cytologie Pathologiques, vol. 30, pp. 303-306, 1982.

[23] G. Naryshkin and E. Redfield, "Malignant cystosarcoma phyllodes of the breast in adolescence, with subsequent pregnancy: report of a case with endocrinologic studies," Obstetrics \& Gynecology, vol. 23, pp. 140-142, 1964.

[24] B. F. Gibbs Jr., R. D. Roe, and D. F. Thomas, "Malignant cystosarcoma phyllodes in a prepubertal female," Annals of Surgery, vol. 167, no. 2, pp. 229-231, 1968.

[25] R. T. Long, A. E. Hesker, and R. E. Johnson, "Surgical management of cystosarcoma phyllodes with a report of 8 cases," Missouri Medicine, vol. 59, pp. 1179-1181, 1962.

[26] H. C. Hoover, A. Trestioreanu, and A. S. Ketcham, "Metastatic cystosarcoma phylloides in an adolescent girl: an unusually malignant tumor," Annals of Surgery, vol. 181, no. 3, pp. 279-282, 1975.

[27] J. F. Ngala Kenda, "Fatal metastatic cystosarcoma Phylloides in a young woman," Archives of Surgery, vol. 118, no. 7, pp. 871$872,1983$.

[28] D. Ogun, O. Gunhan, and H. Goze, "Cystosarcoma phyllodes: a malignant cystosarcoma phyllodes in a 14 year-old girl," The Turkish Journal of Pediatrics, vol. 28, pp. 199-203, 1986.

[29] C. I. C. Turalba, A. M. el Mahdi, and L. Ladaga, "Fatal metastatic cystosarcoma phyllodes in an adolescent female: case report and review of treatment approaches," Journal of Surgical Oncology, vol. 33, no. 3, pp. 176-181, 1986.

[30] J. Schwartz, A. R. Lentini, and A. Woodworth, "Borderline malignant phyllodes tumor in a 12-year-old: applying oncoplastic principles to adolescents," The American Surgeon, vol. 85, no. 5, pp. e252-e253, 2019.

[31] V. Tiwari, V. Mandloi, and H. U. Ghori, "Malignant phyllodes tumor of the breast with isolated brain metastasis: a case report of an intriguing aggressive subtype," Clinical Cancer Investigation Journal, vol. 6, no. 3, pp. 167-170, 2017.

[32] S. R. Lakhani, I. O. Ellis, S. J. Schnitt, P. H. Tan, and M. J. Vijvervan de, Eds., World Health Organization Classification of Tumours of the Breast, International Agency for Research on Cancer (IARC), Lyon, 2012.

[33] F. A. Tavassoli and P. Devilee, Pathology and Genetics of Tumours of the Breast and Female Genital Organs. World Health Organization Classification of Tumours, World Health Organization, Lyon, 2003.

[34] Y. Zhang and C. G. Kleer, "Phyllodes tumor of the breast: histopathologic features, differential diagnosis, and molecular/genetic updates," Archives of Pathology \& Laboratory Medicine, vol. 140, no. 7, pp. 665-671, 2016.
[35] M. Abe, S. Miyata, S. Nishimura et al., "Malignant transformation of breast fibroadenoma to malignant phyllodes tumor: long-term outcome of 36 malignant phyllodes tumors," Breast Cancer, vol. 18, no. 4, pp. 268-272, 2011.

[36] P. B. Gordon, F. A. Gagnon, and L. Lanzkowsky, "Solid breast masses diagnosed as fibroadenoma at fine-needle aspiration biopsy: acceptable rates of growth at long-term follow-up," Radiology, vol. 229, no. 1, pp. 233-238, 2003.

[37] Y. Gao, M. A. Saksena, E. F. Brachtel, D. C. terMeulen, and E. A. Rafferty, "How to approach breast lesions in children and adolescents," European Journal of Radiology, vol. 84, no. 7, pp. 1350-1364, 2015.

[38] B. Y. Tan, G. Acs, S. K. Apple et al., "Phyllodes tumours of the breast: a consensus review," Histopathology, vol. 68, no. 1, pp. 5-21, 2016.

[39] J. Y. Teo, C. S.-J. Cheong, and C. Y. Wong, "Low local recurrence rates in young Asian patients with phyllodes tumours: less is more," ANZ Journal of Surgery, vol. 82, no. 5, pp. 325-328, 2012.

[40] R. Z. Karim, S. K. Gerega, Y. H. Yang et al., "Phyllodes tumours of the breast: a clinicopathological analysis of 65 cases from a single institution," Breast, vol. 18, no. 3, pp. 165-170, 2009.

[41] J. C. Gutierrez, N. Housri, L. G. Koniaris, A. C. Fischer, and J. E. Sola, "Malignant breast cancer in children: a review of 75 patients," The Journal of Surgical Research, vol. 147, no. 2, pp. 182-188, 2008. 